\title{
A topographic event-related potential follow-up study on 'prepulse inhibition' in first and second episode patients with schizophrenia
}

\author{
Stefan Bender ${ }^{1}$, Ulrich Schall ${ }^{1}$, Jörg Wolstein ${ }^{1}$, Ina Grzella ${ }^{1,2}$, Dieter Zerbin ${ }^{1,2}$, \\ Robert D. Oades ${ }^{1,2}$
}

1999 Psychiatry Research (Neuroimaging), 90, 41-53

${ }^{1}$ Biological Psychiatry Research Group, the University Clinics for Adult Psychiatry and Psychotherapy, and for ${ }^{2}$ Child and Adolescent Psychiatry and Psychotherapy, Virchowstr. 174, 45147 Essen, Germany

\begin{abstract}
Dopamine agonists impair and antagonists normalize prepulse inhibition (PPI) of startle and gating of the P50 event-related potential (ERP), but the within-subject effect of treatment on impaired gating in schizophrenia has not been studied.

We report the first results of a longitudinal study using PPI of ERPs as a measure of sensory gating in an auditory Go/No-Go discrimination. After admission and approximately 3 months later, at discharge, 15 patients with schizophrenia performed discrimination between a $1.4 \mathrm{kHz}$ target tone and an $0.8 \mathrm{kHz}$ non-target tone with no prepulse, or with a prepulse at $100 \mathrm{~ms}$ or $500 \mathrm{~ms}$ before either tone. ERPs were recorded from 19 sites. Healthy subjects were studied twice, with 3 months between sessions.

PPI of the P50 peak in the 100-ms condition was reduced in patients on admission. At discharge, decreased negative symptoms correlated with enhanced P50-PPI at fronto-central sites. After treatment increased N100-PPI at centro-temporal sites correlated with fewer positive symptoms. At frontal sites in the $100 \mathrm{~ms}$ condition, the initially small difference of non-target minus target P300 amplitudes increased as negative symptoms decreased.

It is concluded that weak auditory prepulses interfere with early auditory stimulus processing (P50), channel selection (N100) and selective attention (P300). Gating of these stages of processing is impaired in psychotic patients and treatment tends to normalize gating in tandem with improvements of different types of symptoms.
\end{abstract}

Keywords: Sensory gating; Go/No-Go task; Attention; P50; N100; P300; Positive and negative symptoms

\section{Introduction}

Several types of tasks are designed to investigate the effects on the neural processing of information about a salient stimulus exerted by another stimulus that immediately precedes it. One such task is a measure of sensorimotor gating known as prepulse inhibition (PPI) In this task, the effect of a soft prepulse (S1) 80-300 ms before a loud salient tone (S2) is measured in terms of the reduction of the amplitude of the behavioural response of the rodent or human subject_e.g. startle jump or blink; Hoffman and Ison, 1980. This amplitude reduction (inhibition) is less apparent in patients with one of several psychiatric/ neurologic disorders (e.g. schizophrenia, Braff et al., 1978; obsessivecompulsive disorder (OCD), Swerdlow et al., 1993; Schall et al., 1996; Tourette's syndrome, Castellanos et al., 1996; and Huntington's disease, Swerdlow et al., 1995). However, it is difficult to study the effect of experimental manipulations on stages of information processing as, of course, the orienting, attentional and response organization processes are confounded if only a behavioural measure is taken. Nonetheless, in an interesting variant of this task where attention was directed to the prepulse, Dawson et al. (1993) demonstrated that a task-relevant S1 actually enhanced PPI in healthy subjects, but not in patients with 
schizophrenia. Further the startle-PPI task has proved useful, especially in animal studies, for showing that this gating process is modulated by the interactions of a number of neurotransmitters, including dopamine (Swerdlow et al., 1994), serotonin (Sipes and Geyer, 1995; Padich et al., 1996), glutamate (Wan et al., 1995., and $\gamma$-aminobutyric acid (Kodsi and Swerdlow, 1995). In conclusion, PPI may reflect a centrally mediated gating mechanism that regulates sensory, motor, and cognitive operations (Braff and Geyer, 1990).

A second type of task, the conditioningtesting form (CT gating), uses the same stimuli for S1 and S2 with the dependent variable(s) being the size(s) of event-related potential (ERP) components that signify the stages of perceptual and attentional processing of the input from S2 within the first 500 ms (e.g. Nagamoto et al., 1991). The emphasis in this task is on sensory gating, and investigators have used it to show impaired gating by $\mathrm{S} 1$ on the P50 component elicited by S2 in patients with schizophrenia. A third type of task uses a task-relevant S2 (target or non-target) in an auditory Go/No-Go discrimination (Schall et al., 1996, 1997). It combines aspects of the other two task forms (e.g. use of a soft prepulse (S1), but with ERP-dependent measures for S2). As similar effects have been noted for the prepulse on the ERPs and on the eye muscle responses to S2 (Schall et al., 1996), this task may be regarded as providing PPI-like measures of sensory gating on several stages of information processing (P50, N100, $\mathrm{P} 300)$ and signal detection indices of the tasksolving strategies used (Schall and Ward, 1996).

In the PPI-discrimination task, we have shown that in healthy subjects stimulus processing is 'biased' (beta-criterion) towards S2 by a prepulse $100 \mathrm{~ms}$ before S2, as indicated by fewer errors of omission and more false alarms (Schall and Ward, 1996). At frontal recording sites this performance feature correlated with an ERP component, namely the 'prepulse-induced non-target P300-positivity' (PINTP). In contrast, patients with schizophrenia or OCD responded with more errors of omission when S2 was preceded by a prepulse, and they showed a reduced PINTP ampli-tude (Schall et al., 1996).

Recently, we found that the indirect dopamine agonist $d$-amphetamine, which can elicit psychotic symptoms in vulnerable subjects, effectively disrupts the bias towards S2-processing in healthy subjects (Kröner et al., 1998). This disruption was also found to be associated with a reduction of PINTP and a dose-related reduction of $\mathrm{P} 2$ positivity of the ERP elicited by the prepulse. The latter result suggests an involuntary attentional shift towards S1 processing (Michie et al., 1993; Oades et al., 1996) with increasing dopamine release, and resembles the PPI-discrimination deficit reported in patients with schizophrenia or OCD (Schall et al., 1996). Thus, there appears to be a 'window' prior to a contextually important stimulus when a prepulse can alter selective attention via a dopaminergic mechanism, putatively by redirecting or switching the process-ing capacity between stimuli (Oades, 1985). However, the relationship of PINTP to the nature of the symptoms shown by patients with schizophrenia has not been studied. Hence, we here report on the changes of this electro-physiological measure in a longitudinal within-subjects design for patients with schizophrenia (between admission and release) in order to establish if there are changes and whether the measure reflects more a state or a trait feature of information processing in psychosis. Interactions of S1 with the N100 component elicited by S2 have also been described. At inter-stimulus intervals (ISIs) of less than 300 ms, relatively loud pre-stimuli were found to facilitate the N100 response in a passive listening task (Budd and Michie, 1994). In contrast, a pronounced inhibition of N100 was found when a weak prepulse was used $100 \mathrm{~ms}$ before the S2 to which subjects were attending selectively (Schall et al., 1996). These findings indicate that $\mathrm{N} 100$ is an index of sensory processing that varies with prestimulus intensity and selective attention. Thus, a closely preceding loud stimulus increased ERP responses to S2 in a passive presentation, but the processing of a weak S1, on the other hand, captured resources for processing S2 (hence PPI in the 
discrimination). However, this latter gating effect on N100 amplitude may be reduced in patients with schizophrenia: Schall et al. (1997) reported a deficit particularly at lefttemporal recording sites. Here, the focus of our study was on the potential association of acute or positive symptoms with auditory gating deficits (e.g. auditory hallucinations, delusions, CT gating; Freedman et al., 1994. in the PPI-discrimination task-form where clinical improvement between admission and discharge might be expected to be reflected predominantly along the dimension of florid reality distortion.

One research group has consistently reported on impaired CT gating of the P50 ERP component in patients with schizophrenia (e.g. Nagamoto et al., 1989, 1991, 1996). A similar gating impairment in patients with schizophrenia has been confirmed for the PPIdiscrimination task, especially from the left hemisphere (Schall et al., 1997). In the present study we sought to replicate this finding, and to explore the potential relationship to symptom change and neuroleptic responsivity, in view of a report that gating of this component may be sensitive to the clinical response to neuroleptic treatment (Nagamoto et al., 1996). These authors reported increased P50 suppression in six patients respond-ing to clozapine treatment vs. three who were not responding However, this result was obtained at a long ISI ( $500 \mathrm{~ms})$, was not replicated for a shorter ISI (100 ms), was not related to the symptoms expressed and refers to a very small sample. Hence we considered the amount and type of medication administered between PPIdiscrimination measures on admission and at discharge along with the nature of the symptoms that improved as indicators of the type of variable that might contribute to changes in gating during hospitalization.

\section{Methods}

\subsection{Subjects}

Ten male and five female psychotic inpatients (11 first-episode and four second-episode patients) diagnosed according to DSM-IV and ICD-10 criteria and right-handed (Oldfield, 1971) were tested at the start of neuroleptic treatment on admission and again 8-21 weeks later (median=3 months) shortly before discharge from the clinic. The initial diagnosis was confirmed in a 6- month follow-up (ICD10 diagnosis: schizophrenia (F20.x; $n=11$ ), schizophreniform (F23.x; $n=3)$, and schizoaffective (F25.x; $n=1)$ ). Mean age of these patients was $25.4 \pm 7.9$ years. Exclusion criteria included being older than $45 y$-of-age and having head injuries or drug-induced psychosis (according to DSM-IV criteria). Patients were treated with haloperidol ( $n$ at baselines $=5, n$ at re-test $=1$ ), flupenthixol $(3 / 2)$, perazine $(2 / 2)$, risperidone $(1 / 4)$, bromperidol (01), fluphenazine (0/1), chlorprothixene $(0 / 1)$, zotepine $(0 / 1)$. No patient received clozapine. Patients were recorded after receiving up to 3 days' neuroleptic treatment of $0-750 \mathrm{mg}$ (median= $290 \mathrm{mg}$ ) chlorpromazine equivalents daily at baseline (4 patients were drug-naïve) and 235$750 \mathrm{mg}$ (median=404 mg) chlorpromazine equivalents daily at the second test ( 2 patients were drug-free): 4 patients received benzodiazepines at baseline and 2 patients biperidene when tested at follow-up.

There were 8 male and 7 female healthy right-handed control subjects who reported no use of psychotropic medication, no major medical problems and no psychiatric illness in themselves or among first-degree relatives (mean age $=23.9 \pm 5.3$ years). After approval of the protocol by the clinic ethics committee, all subjects consented to participate after being informed of the nature and content of the study. The parents of those under $18 \mathrm{y}$-ofage gave written consent.

\subsection{Clinical ratings}

Patients were rated on the Positive and Negative Syndrome Scale (PANSS; Kay et al., 1992, which includes the Brief Psychiatric Rating Scale) by trained raters (S.B., I.G. and J.W) at both tests.

\subsection{Task}

Auditory stimuli were played bilaterally over headphones (Bayer Dynamics DT 48). The prepulse (S1) was a 0.6 -ms alternating rectangular click at $50 \mathrm{dBSL}$ against a background of white noise (30 dBSL). The second pulse (S2) followed at an ISI of 100 or $500 \mathrm{~ms}$ and consisted of a $0.8-\mathrm{or} 1.4-\mathrm{kHz}$ tone 
( $P=50 \%$ ) at $65 \mathrm{dBSL}$ lasting $50 \mathrm{~ms}$ (rise-fall time of $10 \mathrm{~ms}$ ). Tone and ISI conditions were presented in a Bernoulli sequence of 100 trials per block (no prepulse, $100 \mathrm{~ms}$ and $500 \mathrm{~ms}$ prepulse with $P=33 \%$, respectively) with an inter-trial interval (ITI) of 3-5s. Each subject received 4-6 blocks of trials.

\subsection{Procedure}

The auditory detection threshold of each subject $(0.125-8 \mathrm{kHz})$ was determined immediately before the experiment with an audiometer (Audio-Med BCA3). This allowed individual adjustment of the loudness of the tones for each ear. Subjects relaxed in a reclining soft chair in a small, soundattenuated, air-conditioned and electrically isolated room illuminated indirectly by a $25 \mathrm{~W}$ lamp; subjects could be observed on closedcircuit video. They were asked to make a 2tone discrimination and to respond with a button press with their dominant hand for the high-pitched tone.

\subsection{Recording methods}

The EEG was recorded from 19 tin electrodes (international 10:20 system; Electrocap International) with linked mastoid reference (maximal impedance $2 \mathrm{k} \Omega$ ). The signal was amplified by a Siemens EEG-21 (band pass filter 0.2-70 Hz; amplification $12 \mathrm{k}$ ). EEG epochs were rejected if the vertical or horizontal EOG exceeded $75 \mathrm{mV}$ at any time over the course of a trial (pre-S1 to post-S2, c. $1.6 \mathrm{~s}): 40-50 \%$ of trials were rejected in the control (CON) group and $50-70 \%$ in the schizophrenia spectrum ( $\mathrm{SCH}$ ) group (CON vs. $\mathrm{SCH}: \quad z=-2.4 ; P<0.04)$. These epochs were predominantly rejected as a result of a higher eye-blink rate in the $\mathrm{SCH}$ group. In order to avoid confounding artefacts of frontal ERP measures (e.g. coincident with PINTP), a strict rejection criterion was employed and no EOG correction procedure was performed. Artefact-free recordings (minimum of 20 per stimulus-ISI combination) were digitized at $250 \mathrm{~Hz}$ until $1300 \mathrm{~ms}$ after S1 and referred to a 50 -ms pre-S1 baseline and averaged off-line. The windows for peak measurement were as follows: S2, 30]80 ms (P50); 81-140 ms (N100); 280-400 ms (P300). Peaks in the 500-ms condition were referred to a 50-ms pre-S2 baseline. Those in the 100-ms condition were corrected by subtracting the S1 waveform from the corresponding 500-ms condition in order to separate the P50 component evoked by S2 from the overlapping S1 potential (see Nagamoto et al., 1996).

\subsection{Statistical methods}

Baseline/re-test comparisons of PANSS rating scores and performance measures (i.e. reaction time across pre-pulse conditions) were made with the Friedman Two-Way ANOVA or the Wilcoxon Matched-Pairs Signed Ranks Test, respectively. Group comparisons of these measures were tested with the Mann-Whitney U-Test.

Measures of response bias or criterion (beta) were calculated from hit and false alarm rates in 800-ms post-S2 response windows prior to electrophysiological artefact rejection. Criterion values around 1.0 increase as a liberal response bias (= prepared to make errors of commission to maximise the hit rate) becomes conservative (= prepared to make errors of omission to reduce false alarms).

P50 and N100 amplitudes were anal-ysed at $\mathrm{Cz}$ and P300 amplitudes at Fz electrodes using a multivariate analysis of variance design (MANOVA; exact Wilks $F$ statistics): prepulse interval (3) by target/non-target (2) by re-test (2) by group (2), respectively. Post hoc comparisons of amplitude measures were made with the Wilcoxon Matched-Pairs Signed Ranks Test. Spearman rank correlations were calculated for associations between the changes of symptom and ERP amplitudes recorded from $\mathrm{Fz}, \mathrm{Cz}, \mathrm{T} 3$ and $\mathrm{T} 4$. The statistical rejection criterion was $\alpha<0.05$ (two-tailed probability). Means and standard deviations $( \pm)$ are used as descriptive statistic measures.

\section{Results}

\subsection{Clinical ratings}

BPRS ratings of symptoms improved significantly in the course of neuroleptic treatment (from $55.3 \pm 11.4$ to $35.2 \pm 8.0 ; z=$ 2.6; $P<0.01)$. PANSS ratings showed a marked improvement of positive symptoms (from $24.7 \pm 6.3$ to $12.9 \pm 4.6 ; z=-2.9 ; P<0.01)$ and a moderate improvement of general psychopathology (from $49.0 \pm 9.5$ to $35.1 \pm 7.3$; $z=-2.7 ; \quad P<0.02)$. There was a trend for 
improvement of negative symptoms (from $21.9 \pm 5.9$ to $16.6 \pm 4.3 ; z=-1.8 ; P=0.08)$.

\subsection{Discrimination performance}

Patients responded more slowly and with more errors of omission than healthy subjects (group comparisons: $z=-2.8 ; P<0.01$; Fig. 1 ). Healthy subjects' errors of commission tended to decrease at re-test (baseline vs. re-test: $z=$ -1.9; $P=0.06$ ) while patients significantly improved their hit rate on the second session _baseline vs. re-test: $z=-2.2 ; P<0.05$. Across prepulse conditions, reaction time significantly decreased with increasing prepulse interval in both groups $\left(X^{2}>13.8, d f=2, P<0.01\right)$.

Across prepulse conditions, patients responded with a more conservative bias (e.g. more errors of omission. than healthy subjects (group comparison at baseline: $z=-2.8$; $P<0.01$ ). Patients' beta-criterion changed significantly across pre-pulse conditions in the re-test session $\left(X^{2}=6.3\right.$, d.f. $\left.=2, P<0.05\right)$ and was more liberal in the $100 \mathrm{~ms}$ condition when compared with the no-prepulse condition and the baseline test ( $z>-2.2$; $P<0.05)$, due to more errors of commission (baseline vs. re-test: $z=-2.1 ; P<0.05$ ). There was no significant correlation of the amount of medication administered in chlorpromazine equivalents and reaction time $\left(r_{s}=0.21\right)$.

\subsection{P50 measures}

Mean P50 peak latencies ranged from 48 to $64 \mathrm{~ms}( \pm 5-20)$. No significant latency differences were found between groups, sessions, or across prepulse conditions. Table 1 summarises the amplitudes of the P50 component at $\mathrm{Cz}$. In both groups, target P50 amplitude tended to be larger than non-target amplitudes _target-non-target: $F_{1,28}=4.1$; $P=0.05)$. Peak measures differed significantly across prepulse conditions (interval: $F_{2,27}=$ 39.3; $P<0.0001)$. In healthy subjects, the prepulse in the $100 \mathrm{~ms}$ ISI condition resulted in a non-significant amplitude reduction (3.0 $\pm 2.1 \mu \mathrm{V}$ vs. $3.9 \pm 2.1 \mu \mathrm{V}$ on re-test) and a smaller reduction in the $500 \mathrm{~ms}$ ISI condition $(1.4 \pm 2.1 \mu \mathrm{V}$ vs. $1.7 \pm 2.2 \mu \mathrm{V}$ on re-test); all measures are relative to the no-pulse condition. The baseline-re-test correlation was $r_{s}=0.64(P=0.01)$.

In patients, P50 suppression at base-line was found to be significantly smaller across prepulse conditions than in healthy subjects (group by interval: $F_{2,27}=3.8 ; P<0.04$; Table 1). Re-testing indicated a trend towards an increase of P50-PPI in the $\mathrm{SCH}$ group across pre-pulse conditions (group by interval by retest: $F_{2,27}=2.8 ; P=0.07$. and was found to be maximal in the 100-ms ISI condition (no prepulse vs. $100 \mathrm{~ms}$ prepulse condition: $z=$ 2.1; $P<0.05$; Fig. $2 \mathrm{~A}$ and Fig. 3.. This increase in the $100-\mathrm{ms}$ prepulse condition was significantly correlated with an improvement of negative symptom ratings on PANSS between admission and discharge $\left(r_{s}=-0.60\right.$; $P<0.02)$ and not with medication. admission and discharge.

\section{Table 1}

Mean P50 peak amplitudes ${ }^{\mathrm{a}}(\mu \mathrm{V}$, S.D.; see text for further statistics) recorded at $\mathrm{Cz}$ after the target (T) and non-target (NT) tones in 15 healthy control subjects(CON) and 15 schizophrenic patients(SCH) at baseline and re-test in a no-prepulse condition (no-S1) and in two prepulse conditions with 100- and 500-ms S1-S2 ISIs

\begin{tabular}{llllllll}
\hline & $\begin{array}{l}\text { S1-S2 } \\
\text { interval }\end{array}$ & $\begin{array}{l}\text { Baseline } \\
\text { No-S1 }\end{array}$ & $100 \mathrm{~ms}$ & $500 \mathrm{~ms}$ & $\begin{array}{l}\text { Re-test } \\
\text { No-S1 }\end{array}$ & $100 \mathrm{~ms}$ & $500 \mathrm{~ms}$ \\
\hline \multirow{2}{*}{$\mathrm{CON}$} & $\mathrm{T}$ & $+1.3 \pm 2.0$ & $-2.4 \pm 2.0$ & $-0.7 \pm 2.2$ & $+2.0 \pm 2.3$ & $-2.1 \pm 1.8$ & $+0.5 \pm 2.4$ \\
& $\mathrm{NT}$ & $+0.4 \pm 1.9$ & $-1.9 \pm 2.5$ & $-0.5 \pm 2.4$ & $+0.9 \pm 1.7$ & $-2.9 \pm 2.6$ & $-1.0 \pm 2.1$ \\
& & & & & & & \\
$\mathrm{SCH}$ & $\mathrm{T}$ & $+1.4 \pm 1.2$ & $-0.2 \pm 2.1$ & $+0.3 \pm 2.1$ & $+1.7 \pm 2.6$ & $-0.6 \pm 2.7$ & $-1.2 \pm 2.0$ \\
& $\mathrm{NT}$ & $+1.0 \pm 2.6$ & $-0.3 \pm 2.8$ & $+0.5 \pm 2.5$ & $+1.7 \pm 2.6$ & $-0.3 \pm 2.8$ & $+0.3 \pm 2.6$ \\
\hline
\end{tabular}

${ }^{a}$ Peaks in the 500-ms condition were referred to a 50-ms pre-S2 baseline and those in the 100-ms condition were corrected by subtracting the $\mathrm{S} 1$ waveform from the corresponding 500-ms condition. 


\section{Errors of Commission}

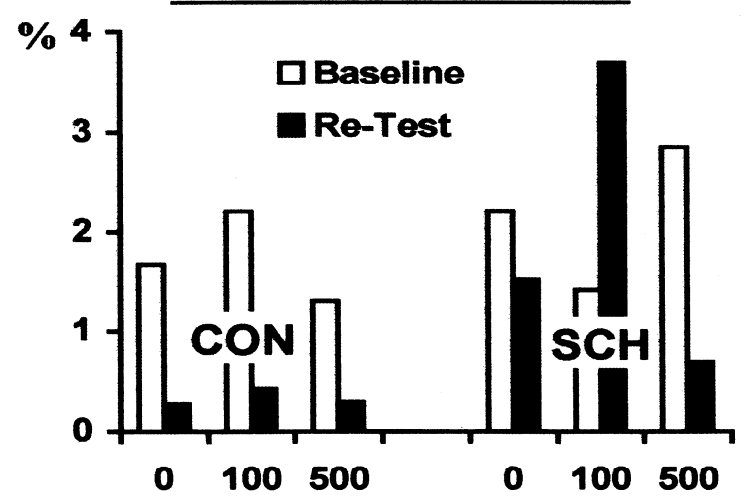

Errors of Omission

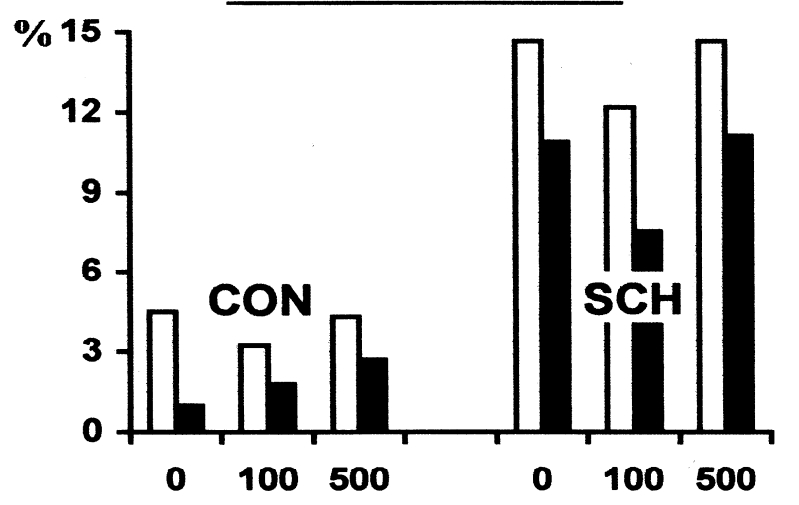

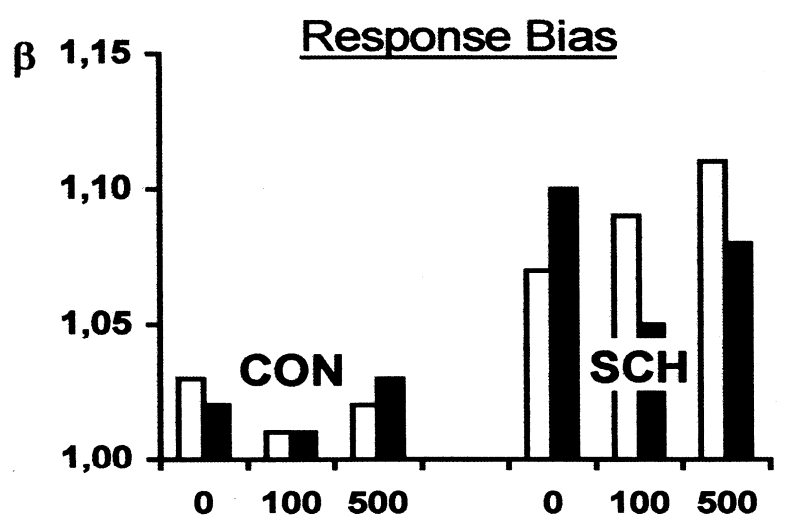

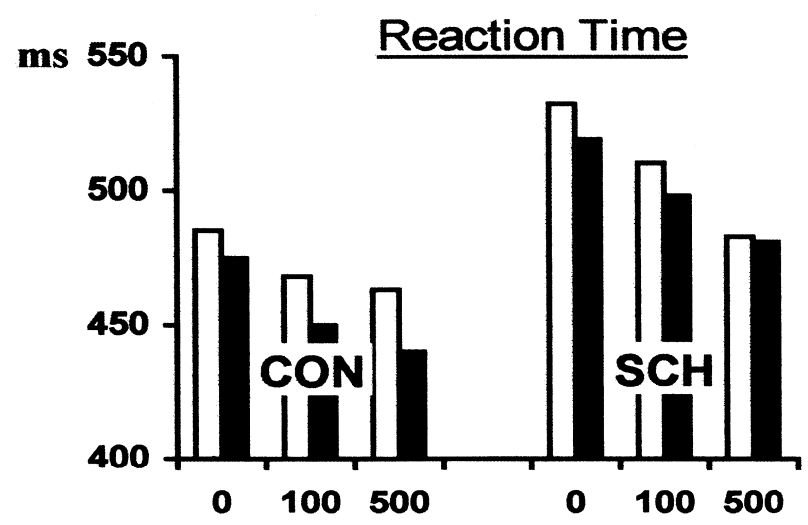

Fig. 1. Errors of commission $( \pm 0.4-5.3 \%)$ and omission $( \pm 1.9-9.7 \%)$, response bias _ b-criterion: $\pm 0.02-0.3)$ and reaction time measures $( \pm 16-135 \mathrm{~ms})$ of 15 schizophrenic patients $(\mathrm{SCH})$ in a nopulse and two prepulse conditions (100 and $500 \mathrm{~ms}$ ISI) at treatment onset and on re-test after 8-21 weeks (median 3 months) of treatment. Performance measures are compared with those of 15 healthy subjects $(\mathrm{CON})$ re-tested after a similar period of time.

\subsection{N100 measures}

N100 peak latencies did not differ significantly between groups, sessions, or across prepulse conditions (range 106-129 ms [ $\pm 16-27])$. In both groups, non-target N100 amplitudes at $\mathrm{Cz}$ were more negative than target $\mathrm{N} 100$ amplitudes (target-non-target: $F_{1,28}=7.6 ; P=0.01 ;$ Table 2). N100-PPI also differed across prepulse conditions in both groups (interval: $F_{2,27}=39.2 ; P=0.001$; see Fig. 4) due to a significant amplitude suppression in the 100 ms prepulse condition (no prepulse vs. $100 \mathrm{~ms}$ pre-pulse condition: $z=-3.7 ; P<$ 0.001). Across conditions, N100 amplitudes of patients were smaller by $1.9 \pm 2.3 \mu \mathrm{V}$ in comparison to healthy subjects (group: $F_{1,28}=$ 6.6; $P<0.02$; Fig. 2A). On re-test there was a non-significant increase of $\mathrm{N} 100$ amplitudes in the $\mathrm{SCH}$ group accompanied by a significant increase of N100-PPI in the 100-ms ISI prepulse condition of $1.1 \pm 2.6 \mu \mathrm{V}$ (N100 PPI base-line vs. re-test: $z=-2.1 ; P<0.05$; Fig. $2 \mathrm{~A}$ ). This change correlated significantly with improvements of positive symptoms (PANSS; Cz: $\left.r_{s}=0.51, P<0.05\right)$ predominantly over the right hemisphere (T4: $r_{s}=0.76 ; P<0.001$ vs. T3: $\left.r_{s}=0.31\right)$. However, no significant association with medication was found. 

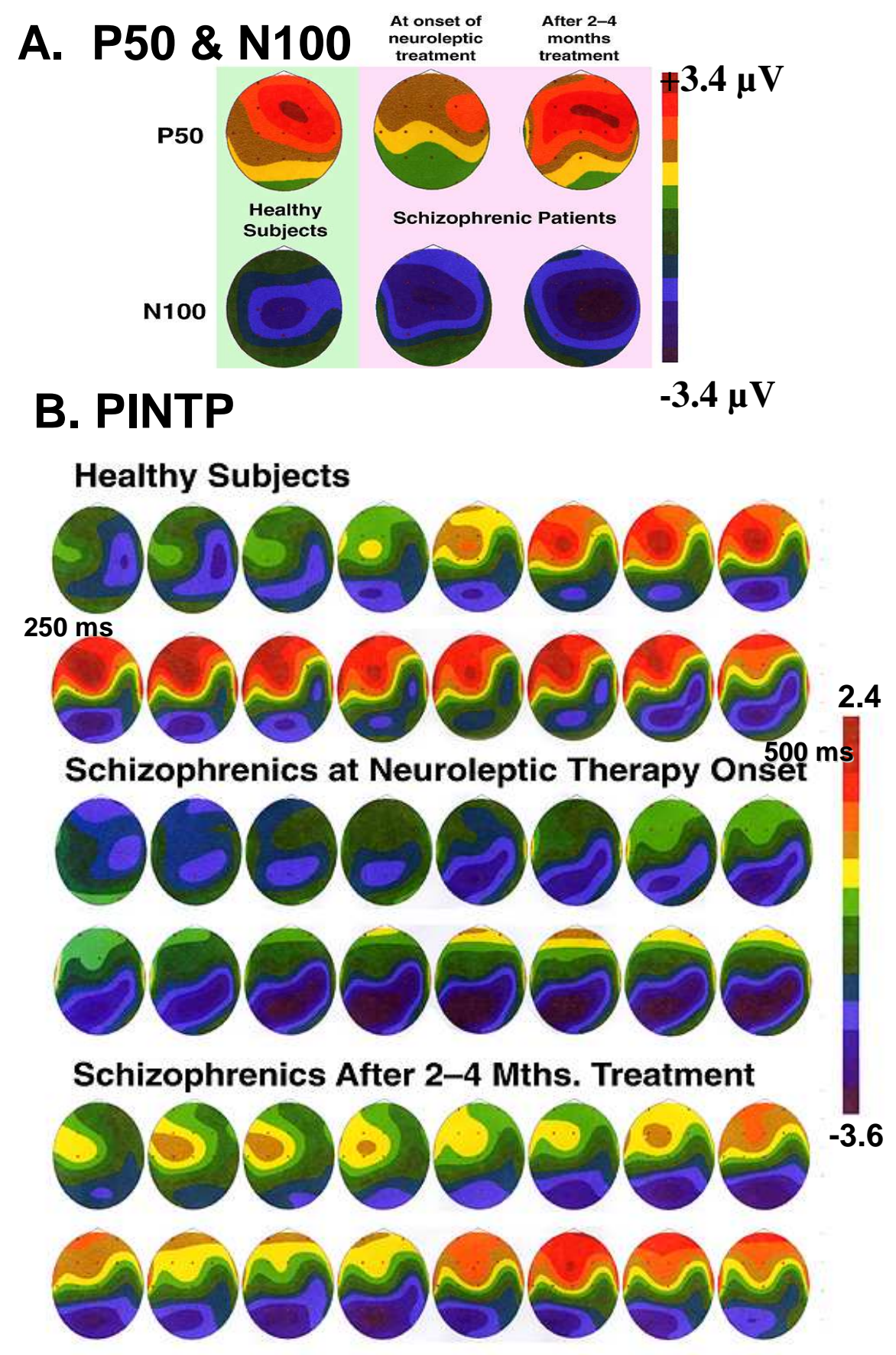

Fig. 2. (A) Averaged topographic mapping of P50 and N100 suppression in the $100 \mathrm{~ms}$ prepulse condition of 15 healthy subjects and 15 schizophrenic patients, the latter at treatment onset and on retest after 8-21 weeks (median $=3$ months) of treatment..

(B) Averaged prepulse-induced non-target positivity (PINTP) of 15 healthy subjects and 15 schizophrenic patients, the latter at treatment onset and on re-test after 8]21 weeks (median $=3$ months) of treatment 


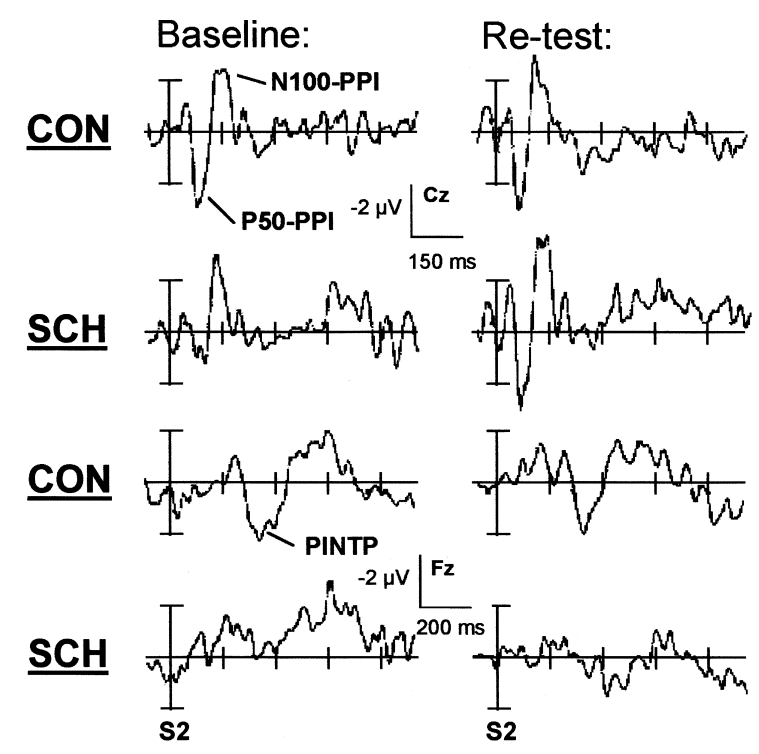

Fig. 3. Difference waves recorded from $\mathrm{Cz}$ (no prepulse minus 100-ms ISI prepulse condition; i.e., PPI) and at Fz (non-target minus target ERPs in the 100-ms prepulse condition; i.e. PINTP) for 15 healthy subjects (CON) and 15 schizophrenic patients $(\mathrm{SCH})$ at treatment onset and on re-test after 8-21 weeks (medians $=3$ months) of treatment.

\subsection{P300 amplitude measures}

Peak P300 latencies ranged from 315 to $353 \mathrm{~ms}( \pm 12-47)$. No significant differences between groups, sessions, and prepulse conditions were found. Non-target P300 amplitudes at $\mathrm{Fz}$ were significantly larger than target P300 amplitudes in both groups, as expected in a Go/No-Go task (target-nontarget: $F_{1,28}=15.7 ; P=0.001$; Table 3). P300 amplitude differed significantly across prepulse conditions (interval: $F_{2,27}=11.4 ; P<$ $0.0001)$, and in particular the difference between target and non-target P300 amplitudes increased when tones were preceded by a prepulse (interval by targetnon-target, i.e. PINTP: $F_{2,27}=21.9 ; P<0.0001$; Fig. 3). PINTP was larger in control subjects than in the $\mathrm{SCH}$ group (group by interval by target-non-target: $F_{2,27}=3.7 ; \quad P<0.04$ ). However, compared with the baseline assessment, patients' PINTP tended to increase at re-test (baseline vs. re-test of P300 non-target minus P300 target amplitudes in $100 \mathrm{~ms}$ prepulse condition: $0.9 \pm 3.1 \mu \mathrm{V} ; z=-$ 1.7, $P<0.1$; Fig. 2B). Increases of PINTP amplitude correlated significantly with improvements in negative symptoms (PANSS; $\left.r_{s}=-0.56 ; P<0.04\right)$ but not with medication.

\section{Discussion}

The present study shows that an increase of inhibitory gating of P50 and N100 ERP components at short S1-S2 intervals to more normal levels occurred during a period of hospitalization accompanied by neuroleptic treatment and was associated with improvements of schizophrenic symptoms. Considering that sources for the P50 and N100 have been reported from structures in the temporal lobe (Reite et al., 1988, 1989), the differential relationship of the two components with negative and positive symptoms, respectively, is consistent with effects on two early but separate stages of processing of auditory information. Thus, the P50 may represent the registration of the arrival of auditory information in the auditory cortex (the 'gate'), while the N100 may represent the subsequent allocation of channels for further processing, important when the stimulus has significance, as in the two-tone discrimination task used here ('resource management'; see Hansen and Hillyard, 1983; Knight et al., 1988; Oades, 1995).

Treatment influenced the amplitudes of the ERP responses discussed, not the reaction times. There were main effects for a decrease of reaction time with the presence of $\mathrm{S} 1$ and the S1-S2 interval and an interaction with diagnosis, where patients with schizophrenia were slower to respond. However, there was no interaction with ISI or treatment. This would seem to preclude alternatives to our explanation of treatment effects on the early stages of processing in terms of motor organization or guessing which tone was the target. There was no clear correlation of the amount of medication administered in chlorpromazine equivalents and the latency of the ERP response nor the reaction time. Indeed the stability of these measures suggests that the patients were able to respond adequately to a 'warning' signal at all times.

The patients investigated here did not exhibit severe negative symptoms; this limits the potential for marked improvement due to the high degree of absolute inter-individual 
Table 2

Mean N100 peak amplitudes ( $\mu \mathrm{V}$, standard deviation; see text for further statistics) recorded at Cz after the target (T) and non-target (NT) tones in 15 healthy control subjects (CON) and 15 schizophrenic patients $(\mathrm{SCH})$ at baseline and re-test in a no-prepulse condition (no-S1) and in two prepulse conditions with 100- and 500-ms S1-S2 ISIs

\begin{tabular}{llllllll}
\hline & S1-S2 & Baseline & & & \multicolumn{2}{l}{ Re-test } & \\
& interval & No-S1 & $100 \mathrm{~ms}$ & $500 \mathrm{~ms}$ & No-S1 & $100 \mathrm{~ms}$ & $500 \mathrm{~ms}$ \\
\hline CON & T & $-9.3 \pm 2.9$ & $-6.9 \pm 2.3$ & $-10.6 \pm 3.8$ & $-8.9 \pm 4.0$ & $-5.8 \pm 3.2$ & $-9.6 \pm 3.3$ \\
& NT & $-10.4 \pm 3.6$ & $-6.7 \pm 2.9$ & $-10.3 \pm 4.1$ & $-10.3 \pm 5.1$ & $-7.0 \pm 3.7$ & $-10.7 \pm 5.2$ \\
& & & & & & & \\
SCH & T & $-8.0 \pm 4.1$ & $-4.2 \pm 2.6$ & $-7.8 \pm 4.1$ & $-8.8 \pm 3.8$ & $-4.1 \pm 2.7$ & $-8.0 \pm 2.5$ \\
& NT & $-7.9 \pm 4.0$ & $-4.8 \pm 2.4$ & $-7.7 \pm 3.6$ & $-9.2 \pm 4.3$ & $-4.8 \pm 3.0$ & $-8.1 \pm 3.4$ \\
\hline
\end{tabular}

Table 3

Mean P300 peak amplitudes ( $\mu$ V, S.D.; see text for further statistics) recorded at Fz after target (T) and non-target (NT) tones in 15 healthy control subjects $(\mathrm{CON})$ and 15 schizophrenic patients (SCH) at baseline and re-test in a no-prepulse condition (no-S1) and in two prepulse conditions with 100- and 500-ms S1-S2 ISIs.

\begin{tabular}{|c|c|c|c|c|c|c|c|}
\hline$\overline{\text { Group }}$ & $\begin{array}{l}\text { S1-S2 } \\
\text { interval }\end{array}$ & $\frac{\text { Baseline }}{\text { No-S1 }}$ & $100 \mathrm{~ms}$ & $500 \mathrm{~ms}$ & $\frac{\text { Re-test }}{\text { No-S1 }}$ & $100 \mathrm{~ms}$ & $500 \mathrm{~ms}$ \\
\hline$\overline{\mathrm{CON}}$ & $\begin{array}{l}\text { T } \\
\text { NT }\end{array}$ & $\begin{array}{l}4.8 \pm 3.0 \\
4.5 \pm 3.0\end{array}$ & $\begin{array}{l}2.5 \pm 2.8 \\
3.7 \pm 3.1\end{array}$ & $\begin{array}{l}1.9 \pm 2.4 \\
6.7 \pm 2.8\end{array}$ & $\begin{array}{l}6.0 \pm 3.6 \\
7.1 \pm 3.6\end{array}$ & $\begin{array}{l}4.4 \pm 2.6 \\
5.6 \pm 3.3\end{array}$ & $\begin{array}{l}3.0 \pm 3.5 \\
7.7 \pm 2.7\end{array}$ \\
\hline $\mathrm{SCH}$ & $\begin{array}{l}\text { T } \\
\text { NT }\end{array}$ & $\begin{array}{l}5.4 \pm 5.0 \\
5.8 \pm 4.3\end{array}$ & $\begin{array}{l}3.8 \pm 3.8 \\
2.9 \pm 3.2\end{array}$ & $\begin{array}{l}2.6 \pm 3.4 \\
4.8 \pm 3.4\end{array}$ & $\begin{array}{l}4.9 \pm 4.0 \\
5.6 \pm 4.0\end{array}$ & $\begin{array}{l}3.9 \pm 2.8 \\
3.9 \pm 3.0\end{array}$ & $\begin{array}{l}3.3 \pm 3.8 \\
5.2 \pm 3.8\end{array}$ \\
\hline
\end{tabular}
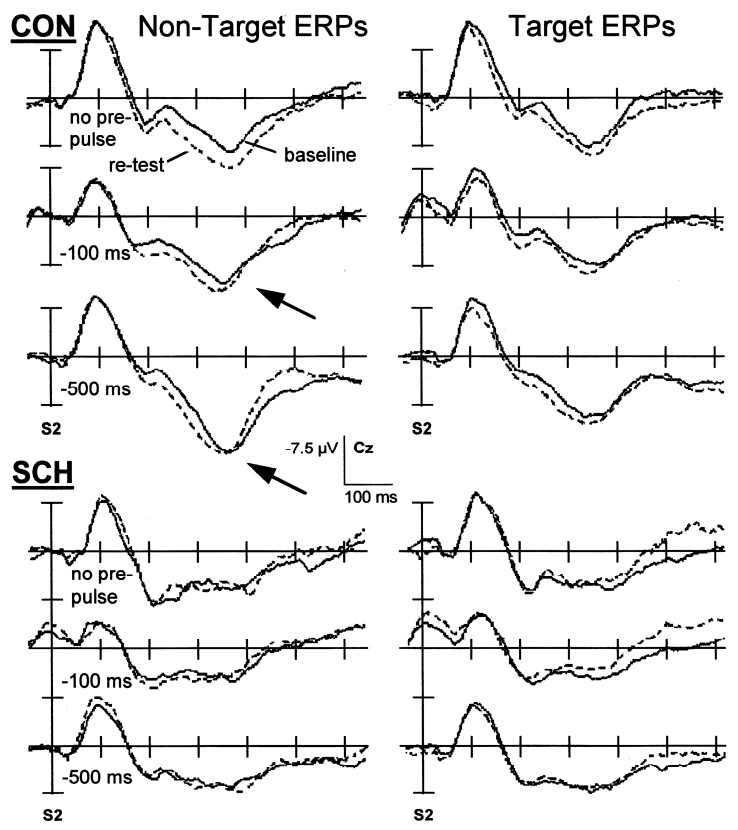

Fig. 4. Event-related potential recordings from $\mathrm{Cz}$ elicited by target and non-target tones in an auditory Go/No-Go discrimination task from 15 healthy subjects (CON) and 15 schizophrenic patients $(\mathrm{SCH})$ at treatment onset and on re-test after 8-21 weeks (median $=3$ months) of treatment. Note prepulse-induced positivity of non-target P3s (PINTP) in healthy subjects (arrows). variability. Thus, the increase of P50 suppression in the $\mathrm{SCH}$ group (see Table 1: group by interval by re-test interaction: $P=0.07$ ) and the improvement of negative symptoms ratings were not found to be significant when assessed independently. Nonetheless, there was a notable trend for an improvement of negative symptoms (25\% reduction; $P=0.08$ ), and this improvement was associated with increased P50 suppression at frontal recording sites and at short S1-S2 intervals when the relative differences between sessions were correlated. These are less confounded by inter-individual variability. (This point also applies to N100 and PINTP correlations with symptom ratings). This association may have been facilitated by antidopaminergic treatment in view of ERP disturbances induced by the psychotomimetic amphetamine in PPI discrimination (Kröner et al., 1998) and improvements of CT gating claimed after neuroleptic treatment (Nagamoto et al., 1996). Animal studies have also emphasised the disruptive effects on PPIstartle of too much serotonergic and dopaminergic activity (Swerdlow et al., 1994; Sipes and Geyer, 1995; Padich et al., 1996), 
and human work with ERPs following CT gating has emphasised the nicotinic and noradrenergic systems in the hippocampal complex (Freedman et al., 1994). Reports from animal studies conflict over the role of the frontal cortex and mesocortical dopamine in PPI of the startle response (lesion-induced disruption, Bubser and Koch, 1994) vs. marginal effects (Swerdlow et al., 1986). However, damage to temporal lobe structures that contain generators for both P50 and N100 does affect CT gating (Knight et al., 1988; Reite et al., 1988; Freedman et al., 1994).

ERPs sensitive to stimulus deviance with similar early latencies have been recorded from temporal lobe structures, such as the hippocampus, in animals (Ruusuvirta et al., 1995). In addition, the mismatch negativity response to stimulus deviance is absent if tones are presented at ISIs of $100 \mathrm{~ms}$, as used in the present study (Winkler and Näätänen, 1992). Mismatch negativity is also reported to have a source in the auditory temporal cortex in humans and is re-corded from more lateral and temporal sites in schizophrenic patients than in healthy subjects (Oades, 1995; Oades et al., 1997). These results underscore the potential of the temporal lobe for media-ting the impairments and treatment-related improvements of the early ERPs described.

The frontal and temporal loci for changes of PPI-discrimination expression reported in schizophrenic patients might also have been predicted on the basis of other clinical reports. Thus, schizophrenic patients who perform poorly on the Wisconsin Card-Sorting Test of frontal function were also those who showed a major loss of startle-PPI (Butler et al., 1990). Indeed the topographic lateralization we have illustrated (see Schall et al., 1997) is consistent with the topography of processing negativity, an ERP index of attentional function (c. 150$250 \mathrm{~ms}$ post-stimulus). This index has an impaired right-frontal-left temporal distribution in non-paranoid schizophrenic patients vs. the opposite pattern in paranoidhallucinatory patients (Oades et al., 1994). Further-more, decreased amplitudes of later attention-related ERPs (P300) in patients with schizophrenia have been directly related to the atrophy shown on MRI scans of the left superior temporal gyrus (McCarley et al., 1993). Thus, it seems likely that there may be an unusual functional and structural asymmetry in the temporal lobes that is likely to be reflected in PPI-discrimination measures. Freedman et al. (1994) even suggested that a lack of PPI might model the way that trivial information could become the source of information for hallucinations and delusions.

In the present study, improvements in positive symptoms were correlated with the increase of $\mathrm{N} 100$ gating from right temporal sites. In healthy subjects such gating is usually found to be maximal over the right hemisphere (Schall et al., 1997). Yet, at the start of treatment, schizophrenic patients already exhibited a marked suppression of N100. Perhaps gating of excitatory ERP components, such as the N100, is more sensitive to early dopamine blockade. Thus, an increase of N100-gating in the course of neuroleptic treatment, to a level above that recorded in healthy subjects, suggests that clinical improvement is achieved at the cost of introducing an imbalance of excitatory and inhibitory processes in the right-temporal lobe to compensate for the contralateral impairment (McCarley et al., 1993). This pre-diction could be addressed by comp-aring records from the acutely ill with those from patients in a later residual state, stabilized on medication. Such a long-term study is in progress. However, since the relationship of conventional surface recording with underlying sources of N100-PPI changes is equivocal, anatomical structures should be determined by high-resolution source analysis or magnetoencephalographic recordings, for instance. Thus, interpretations regarding source-surface relationships on the present database have to be made cautiously.

Late stages of controlled information processing are indexed by the P300 component. In the context of an auditory discrimination task, P300 is an index of attention-guided performance (Grillon et al., 1991). An increase of PINTP, together with an increasingly more liberal response bias in the 100-ms ISI condition, was also associated with improvements of negative symptoms. This 
suggests that there was enhanced attentional control of differential stimulus processing, and in particular, processing of the 'no-go' stimulus was facilitated. As a result the patients' hit rate improved.

The Go/No-Go procedure can be view-ed as a task where performance is also a measure of alternation flexibility and where impairments have been attributed to frontal lobe dysfunction (Sullivan et al., 1993) and associated with negative symptoms in schizophrenia (Liddle and Morris, 1991). The increase of PINTP, with a frontal topography, in this context can be conceptualised as a neurophysiological sign for improved resource allocation to task-relevant stimuli. Processing capabilities appear to have been improved by a more efficient management of resources. The question of whether current forms of treatment are capable of impeding the deterioration of limited resources or reinstating their efficiency to a level that can be maintained after treatment can only be answered with a long-term prospective study. Such a study, in progress, should be able to confirm the proposal of this report that a prepulse can interfere with early automatic and later controlled sensory processing and that both impairments may be amenable to treatment.

\section{Acknowledgements}

We are grateful to Rita Frantska, Jutta Haverkorn, Mark Pearson, and Robert Windelschmitt for technical assistance, to Dr. Philip Ward and Dr. Sally Andrews for discussion and to the Deutsche Forschungsgemeinschaft (DFG Scha 628r4-1) for financial support. Preliminary results were presented on the 8th Winter Work-shop on Schizophrenia (Bender et al., 1996) where S.B. received a Young Scientist Award.

\section{References}

Bender, S., Wolstein, J., Butorac, M., Grzella, I., Ortmann, K., Zerbin, D., Schall, U., Oades, R.D., 1996. Therapy monitoring with prepulse inhibition in an active auditory discrimination in patients with schizophrenia. Schizophrenia Research 18, 224.
Braff, D.L., Geyer, M.A., 1990. Sensory motor gating in schizophrenia: human and animal model studies. Archives of General Psychiatry 47, 181-188.

Braff, D.L., Stone, C., Callaway, E., Geyer, M., Glick, I., Bali, L., 1978. Prestimulus effects on human startle reflex in normals and schizophrenics. Psychophysiology 15, 339-343.

Bubser, M., Koch, M., 1994. Prepulse inhibition of the acoustic startle response of rats is reduced by 6-hydroxydopamine lesions of the medial prefrontal cortex. Psychopharmacology 113, 487-492.

Budd, T.W., Michie, P.T., 1994. Facilitation of the N100 peak of the auditory ERP at short stimulus intervals. NeuroReport 5, 2513-2516.

Butler, R.W., Jenkins, M.A., Geyer, M.A., Braff, D.L., 1990. Wisconsin Card-Sorting deficits and diminished sensorimotor gating in a discrete sub-group of schizophrenic patients. In: Tamminga, C.A., Schulz, S.C. (Eds.), Schizophrenia. Raven Press, New York.

Castellanos, F.X., Fine, E.J., Kaysen, D., Marsh, W.L., Rapoport, J.L., Hallet, M., 1996. Sensorimotor gating in boys with Tourette's syndrome and ADHD: preliminary results. Biological Psychiatry 39, 33-41.

Dawson, M.E., Hazlett, E.A., Filion, D.L., Nuechterlein, K.H., Schell, A.M., 1993. Attention and schizophrenia: impaired modulation of the startle reflex. Journal of Abnormal Psychology 102, 633-641.

Freedman, R., Adler, L.E., Bickford, P., Byerley, W., Coon, H., Cullum, C.M., Griffith, J.M., Harris, J.G., Leonard, S.S., Miller, C., MylesWorsley, M., Nagamoto, H.T., Rose, G., Waldo, M., 1994. Schizophrenia and nicotinic receptors. Harvard Review of Psychiatry 2, 179-192.

Grillon, C., Ameli, R., Courchesne, E., Braff, D.L., 1991. Effects of task relevance and attention on P3 in schizophrenic patients. Schizophrenia Research 4, 11-21.

Hansen, J.C., Hillyard, S.A., 1983. Selective attention to multidimensional auditory stimuli. Journal of Experimental Psychology (Human Perception and Performance) 9, 1-19.

Hoffman, H.S., Ison, J.R., 1980. Reflex modification in the domain of startle: I. some empirical findings and their implications for how the nervous system processes sensory input. Psychology Reviews 87, 172-189. 
Kay, S.R., Opler, L.A., Fiszbein, A., 1992. Positive and Negative Syndrome Scale (PANSS). MultiHealth System Inc., Toronto, Canada.

Knight, R.T., Scabini, D., Woods, D.L., Clayworth, C., 1988. The effects of lesions of superior temporal gyrus and inferior parietal lobe on temporal and vertex components of the human AEP. Electroencephalography and Clinical Neurophysiology 70, 497-509.

Kodsi, M.H., Swerdlow, N.R., 1995. Prepulse inhibition in the rat is regulated by ventral and caudo-dorsal striato-pallidal circuitry. Behavioral Neuroscience 109, 912-928.

Kröner, S., Schall, U., Catts, S.V., Ward, P.B., 1998. Prepulse inhibition (PPI) of event-related potentials (ERPs) in an auditory discrimination is impaired in normal subjects after amphetamine: support for a model of schizophrenia. Schizophrenia Research 29, 120-121.

Liddle, P.F., Morris, D.L., 1991. Schizophrenic syndromes and frontal lobe performance. British Journal of Psychiatry 158, 340-345.

McCarley, R.W., Shenton, M.E., O'Donnell, B.F., Faux, S.F., Kikinis, R., Nestor, P.G., Jolesz, F.A., 1993. Auditory P300 abnormalities and left superior temporal gyrus volume reduction in schizophrenia. Archives of General Psychiatry 50, 190-197.

Michie, P.T., Solowij, N., Crawford, J.M., Glue, L.C., 1993. The effects of between-source discriminability on attended and non-attended auditory ERPs. Psychophysiology 30, 205-220.

Nagamoto, H.T., Adler, L.W., Waldo, M.C., Freedman, R., 1989. Sensory gating in schizophrenics and normal controls: effects of changing the stimulation interval. Biological Psychiatry 25, 549-561.

Nagamoto, H.T., Adler, L.W., Waldo, M.C., Griffith, J., Freedman, R., 1991. Gating of auditory response in schizophrenics and normal controls: effects of recording sites and stimulation interval on the P50 wave. Schizophrenia Research 4, 31-40.

Nagamoto, H.T., Adler, L.W., Hea, R.A., Griffith, J.M., McRae, A., Freedman, R., 1996. Gating of auditory P50 in schizophrenics: unique effects of clozapine. Biological Psychiatry 40, 181-188.

Oades, R.D., 1985. The role of noradrenaline in tuning and dopamine in switching between signals in the CNS. Neuroscience and Biobehavioral Reviews 9, 261-283.
Oades, R.D., 1995. Connections between studies of the neurobiology of attention, psychotic processes and event related potentials. Electroencephalography and Clinical Neurophysiology 44, 428-438.

Oades, R.D., Zerbin, D., Eggers, C., 1994. Negative difference ( $\mathrm{Nd}$ ) an ERP marker of stimulus relevance: different lateral asymmetries for paranoid and non-paranoid schizophrenics. Pharmacopsychiatry 27, 65-67.

Oades, R.D., Dittmann-Balcar, A., Schepker, R., Eggers, C., Zerbin, D., 1996. Auditory eventrelated potentials (ERPs) and mismatch negativity (MMN) in healthy children and those with attention-deficit or Tourette/tic symptoms. Biological Psychology 43, 163-185.

Oades, R.D., Dittmann-Balcar, A., Zerbin, D., Grzella, I., 1997. Impaired attentiondependent augmentation of MMN in nonparanoid vs. paranoid schizophrenic patients: a comparison with obsessive-compulsive disorder and healthy subjects. Biological Psychiatry 41, 1196-1210.

Oldfield, R.C., 1971. The assessment and analysis of handedness: the Edinburgh Inventory. Neuropsychologia 9, 97-113.

Padich, R.A., McCloskey, T.C., Kehne, J.H., 1996. 5-HT modulation of auditory and visual sensorimotor gating: II. Effects of the 5-HT2A antagonist MDL 100,907 on disruption of sound and light prepulse inhibition produced by 5-HT agonists in Wistar rats. Psychopharmacology 124, 107-116.

Reite, M., Teale, P., Zimmerman, J., Davis, K., Whalen, J., Edrich, J., 1988. Source origin of a $50 \mathrm{msec}$ latency auditory evoked field component in young schizophrenic men. Biological Psychiatry 24, 495-506.

Reite, M., Teale, P., Goldstein, L., Whalen, P., Linnville, S., 1989. Late auditory magnetic sources may differ in the left hemisphere of schizophrenic patients: a preliminary report. Archives of General Psychiatry 46, 565-572.

Ruusuvirta, T., Korhonen, T., Penttonen, M., Arikoski, J., 1995. Hippocampal evoked potentials to pitch deviances in an auditory oddball situation in the rabbit: no human mismatch-like dependence on standard stimuli. Neuroscience Letters 185, 123-126.

Schall, U., Ward, P.B., 1996. 'Prepulse inhibition' facilitates a liberal response bias in an auditory discrimination task. NeuroReport 7, 652-656. 
Schall, U., Schön, A., Zerbin, D., Eggers, C., Oades, R.D., 1996. Event-related potentials during an auditory discrimination with prepulse inhibition in patients with schizophrenia or obsessive-compulsive disorder and healthy subjects. International Journal of Neuroscience 84, 15-33.

Schall, U., Schön, A., Zerbin, D., Bender, S., Eggers, C., Oades, R.D., 1997. A left temporal lobe impairment of auditory information processing in schizophrenia: an event-related potential study. Neuroscience Letters $229,25-$ 28.

Sipes, T.E., Geyer, M.A., 1995. DOI disrupt-ion of prepulse inhibition of startle in the rat is mediated by $5-\mathrm{HT} 2 \mathrm{~A}$ and not by $5-\mathrm{HT} 2 \mathrm{C}$ receptors. Behavioral Pharmacology 6, 839842.

Sullivan, E.V., Mathalon, D.H., Zipursky, R.D., Kersteen-Tucker, Z., Knight, R.T., Pfefferbaum, A., 1993. Factors of the Wisconsin Card Sorting Test as measures of frontal-lobe function in schizophrenia and in chronic alcoholism. Psychiatry Research 46, 175-199.

Swerdlow, N.R., Braff, D.L., Geyer, M.A., Koob, G.F., 1986. Central dopamine activity in rat mimics abnormal acoustic startle response in schizophrenics. Biological Psychiatry 21, 23-33.

Swerdlow, N.R., Benbow, C.H., Zisook, S., Geyer, M.A., Braff, D.L., 1993. A preliminary assessment of sensorimotor gating in patients with obsessive compulsive disorder. Biological Psychiatry 33, 298-301.

Swerdlow, N.R., Braff, D.L., Taaid, N., Geyer, M.A., 1994. Assessing the validity of an animal model of deficient sensorimotor gating in schizophrenic patients. Archives of General Psychiatry 51, 139-154.

Swerdlow, N.R., Paulsen, J., Braff, D.L., Butters, N., Geyer, M.A., Swenson, M.R., 1995. Impaired prepulse inhibition of acoustic and tactile startle in patients with Huntington's disease. Journal of Neurology, Neurosurgery and Psychiatry 58, 192-200.

Wan, F.J., Geyer, M.A., Swerdlow, N.R., 1995. Presynaptic dopamine-glutamate interactions in the nucleus accumbens regulate sensorimotor gating. Psychopharmacology 120, 433-441.

Winkler, I., Näätänen, R., 1992. Event-related potentials in auditory backward recognition masking: a new way to study the neurophysiological basis of sensory memory in humans. Neuroscience Letters 140, 239-242. 